\title{
Aluminum Nanocrystals
}

Michael J. McClain ${ }^{1,6}$, Andrea E. Schlather ${ }^{1,6}$,Emilie Ringe ${ }^{1,2,6}$, Nicholas S. King ${ }^{3,6}$, Lifei Liu ${ }^{3,6}$, Alejandro Manjavacas ${ }^{3,6}$, Mark. W. Knight ${ }^{4,6}$, Ish Kumar ${ }^{1}$, Kenton Whitmire ${ }^{1}$, Henry O. Everitt ${ }^{7}$, Peter Nordlander ${ }^{3,4,6}$, Naomi J. Halas ${ }^{1-6}$

${ }^{1}$ Department of Chemistry, ${ }^{2}$ Department of Materials Science and Nanoengineering, ${ }^{3}$ Department of Physics and Astronomy, ${ }^{4}$ Department of Electrical and Computer Engineering,

${ }^{5}$ Department of Bioengineering, ${ }^{6}$ Laboratory for Nanophotonics, Rice University, Houston, Texas, USA. ${ }^{7}$ Army Aviation and Missile RD\&E Center at Redstone Arsenal, AL, USA.

KEYWORDS Plasmon, UV, aluminum, nanocrystal, synthesis

\begin{abstract}
We demonstrate the facile synthesis of high purity aluminum nanocrystals over a range of controlled sizes from $70 \mathrm{~nm}$ to $220 \mathrm{~nm}$ diameter, with size control achieved through a simple modification of solvent ratios in the reaction solution. The monodisperse, icosahedral and trigonal bipyramidal nanocrystals are air-stable for weeks, due to the formation of a 2-4 nm thick passivating oxide layer on their surfaces. We show that the nanocrystals support sizedependent ultraviolet and visible plasmon modes, providing a far more sustainable alternative to gold and silver nanoparticles currently in widespread use.
\end{abstract}


Noble metal nanoparticles are used extensively for applications such as photocatalysis or biological and chemical sensing. ${ }^{1-8}$ Interest in these nanoparticles has been based largely on their vivid optical properties, which are due to their collective electronic resonances, known as surface plasmons. Exquisite size and shape control has been achieved in the synthesis of noble metal nanoparticles such as gold, silver, and platinum, but the intrinsic properties and high cost of these noble metals present significant limitations for large-scale use. ${ }^{7,9,10}$ Gold nanoparticles have been of interest in applications involving visible and near-infrared light, and while the plasmon resonance of silver can also be excited at wavelengths that extend into the ultraviolet region of the spectrum, its conversion from silver to silver oxide limits its long-term stability. The UV region $(\lambda=100 \mathrm{~nm}-400 \mathrm{~nm})$ is of great interest in numerous applications; for example, most organic molecules have a strong absorption in the ultraviolet, allowing for ultrasensitive chemical sensing. Short wave UV light $(\lambda<280 \mathrm{~nm})$ is capable of breaking organic bonds, which is key to sterilization, remediation, and photocatalysis applications.

Aluminum is the most abundant metal in the earth's crust and can support a UV plasmon resonance. $^{3-5,7}$ Recent research on lithographically-fabricated aluminum nanostructures demonstrated that the plasmon resonance is highly sensitive to oxygen content, redshifting and attenuating the plasmon resonance with increasing oxygen content. ${ }^{4}$ Chemical methods for the synthesis of Aluminum nanoparticles have involved the thermal decomposition of an aluminum hydride with a titanium catalyst, but size and shape control have proven to be problematic with this approach. ${ }^{11-19}$ In this letter we report the facile synthesis of highly regular, faceted aluminum nanocrystals with controllable nanocrystal diameters ranging from 70 to greater than $200 \mathrm{~nm}$. The optical properties of individual Al nanocrystals were measured using darkfield spectroscopy, cathodoluminsence (CL), and electron-energy loss spectroscopy, which clearly show the size- 
dependent, continuous tuning of the optical resonance from ultraviolet to the visible region of the spectrum with increasing nanocrystal diameter. The Al nanocrystals are terminated with a selflimiting, 2-4 nm oxide layer, which imparts stability: an important property that can ultimately enable their use in many different types of applications.

The Aluminum nanocrystals were synthesized by adding a capping agent to the reaction shown in Figure 1a. The nanocrystals shown were synthesized using Schlenk line and glove box techniques. All solvents and reagents were purchased from Sigma Aldrich and distilled before use. 1,4-dioxane and Tetrahydrofuran (THF) were added to a dry Schlenk flask under inert atmosphere. Varying volume fractions of THF and 1,4-dioxane were added to equal $25 \mathrm{~mL}$ of solvent. The flask was heated in a $40^{\circ} \mathrm{C}$ oil bath. While the solvent was stirring, $6.5 \mathrm{~mL}$ of $0.5 \mathrm{M} \mathrm{N}, \mathrm{N}$-dimethylethylamine alane was added. $0.5 \mathrm{~mL}$ of a $3.3 \mathrm{mM}$ solution of titanium (IV) isopropoxide in toluene was added rapidly via syringe. After 2.5 minutes, $0.5 \mathrm{~mL}$ of a $250 \mathrm{mM}$ solution of oleic acid in 1,4-dioxane was added rapidly via syringe. The reaction proceeded for 2 hours. The reaction was removed from the heat source and stirred at room temperature for one hour. Vacuum was applied to the solution to remove volatile byproducts. The particles were then cleaned with centrifugation and sonication in dry THF to remove any unreacted alane, followed by three cycles in isopropanol. Isopropanol helps separate the particles from the capping agent. An oxide layer forms in this process which allows the particles to be stable for weeks (Figure S2). The particles were analyzed with X-ray photoelectron spectroscopy to confirm they are aluminum. 


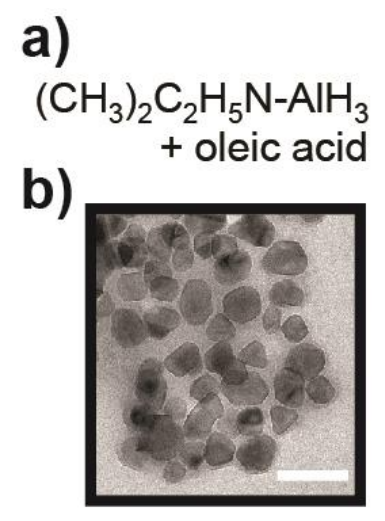

0
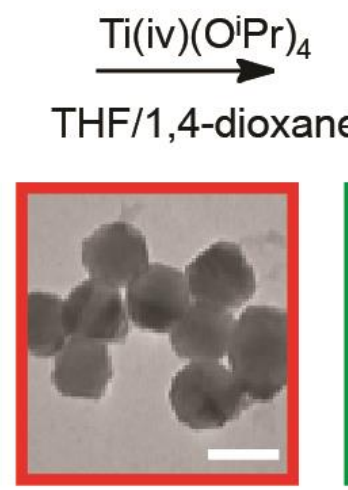

0.5

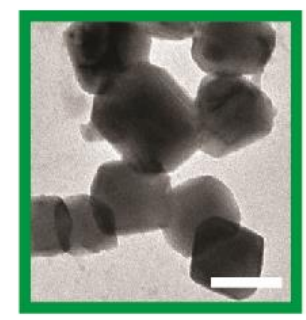

0.6

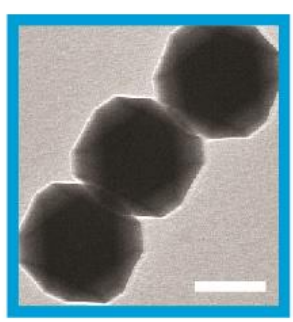

0.8

Figure 1. Size control of aluminum nanocrystals. Increasing the fraction of tetrahydrafuran (THF) in a 1,4-dioxane/THF solution yields larger nanocrystals. a) Reaction scheme for synthesis of aluminum nanocrystals. b) Representative TEM images from synthesis with $0,0.2$, 0.5 , and $0.8 \mathrm{THF}$ volume fractions in a THF/1,4-dioxane solution (from left to right) (Scale bar $=$ $100 \mathrm{~nm})$.

The ratios of THF and dioxane are vital in controlling the sizes of the aluminum nanocrystals obtained by this approach, however their precise role is elusive. We did not observe size or shape control when we varied capping agent concentration, which was also observed organic phase synthesis of indium nanocrystals. ${ }^{20}$ When toluene was substituted for dioxane (toluene has a similar viscosity to THF but a dielectric constant similar to dioxane) the resulting particles had the same size and shape as with the same ratio of THF/dioxane. These results suggest that particle size is more directly related to the dielectric properties of the solution and not to its viscosity.

Histograms of the particles represented in figure 1 were generated by measuring the sizes of 200 particles from each reaction of a given volume fraction (Figure S1). The size 
distributions corresponding to each volume fraction from figure $1 \mathrm{~b}$ (left to right) are $55 \pm 11 \mathrm{~nm}$, $117 \pm 11 \mathrm{~nm}, 152 \pm 35 \mathrm{~nm}$, and $223 \pm 32 \mathrm{~nm}$. Several shapes are present and we observed no change in the percentage of each shape for particles made with different fractions of THF. There are approximately $30 \%$ truncated trigonal bipyramids, $30 \%$ octahedra, $10 \%$ icosahedra, and the remaining $30 \%$ are mixed irregular crystals.
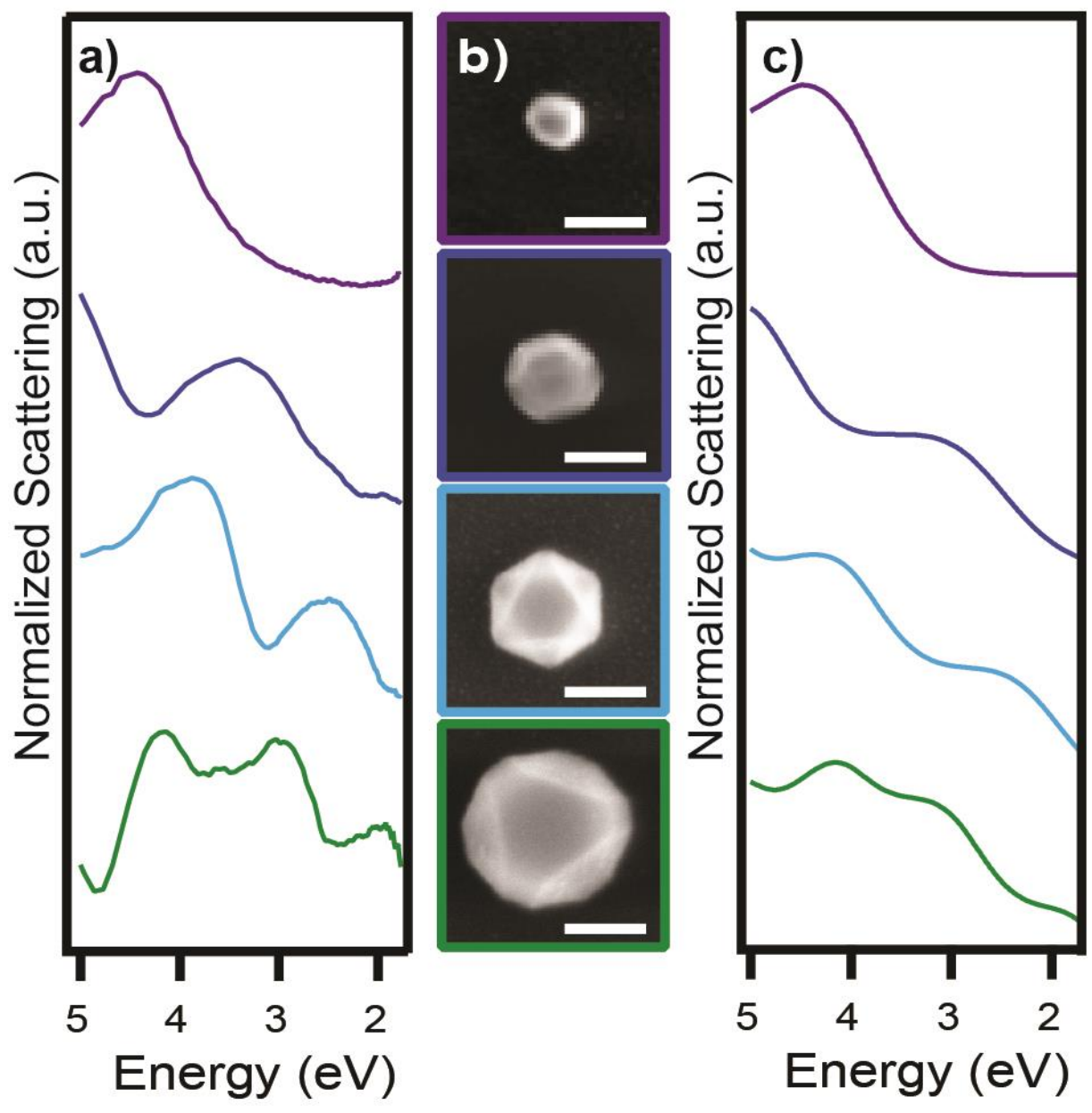

Figure 2. Dark-field spectroscopy of single aluminum nanocrystals. a) Experimental darkfield spectra of 80, 120, 155 and $220 \mathrm{~nm}$ nanocrystals (top to bottom) (Scale bar $=100 \mathrm{~nm}$ ). b) SEM images of respective particles. c) Theoretical calculation of spectra obtained using Mie theory, assuming spherical nanoparticles coated with a $3 \mathrm{~nm}$ oxide layer in a homogeneous dielectric environment. 
The optical properties of individual nanocrystals were obtained using a custom-built UVvisible dark field microscope (Figure 2). For the dark field measurements, a solution of aluminum nanocrystals was spin-coated onto a quartz substrate, and an SEM image of each nanocrystal was obtained following the measurement of its optical properties. The scattering spectrum of a series of nanocrystals of increasing size is shown in Figure 2a. In each case, the spectrum is dominated by the dipolar plasmon mode of the nanocrystal, which redshifts with increasing nanocrystal diameter. For nanocrystal sizes of 120 and $150 \mathrm{~nm}$, we also observe a higher energy quadrupole mode at 5 and $4 \mathrm{eV}$, respectively. In this size range, the nanocrystal diameter is nearly half the size of the plasmon resonant wavelength, which results in significant optical phase retardation, increasing the magnitude of the higher order modes observed in the scattering spectrum. The scanning electron microscope (SEM) images in Figure $2 b$ are representative particles corresponding to each spectrum in Figure 2a.

The experimental dark field spectra were compared with theoretical calculations obtained using Mie theory for similarly sized particles. ${ }^{21}$ Specifically, we model the aluminum nanoparticles as spheres coated by a $3 \mathrm{~nm}$ oxide layer sitting in an homogeneous environment with a dielectric function chosen to account for the effect of the substrate. Using the same illumination and collection conditions as in the experiment, we obtain theoretical spectra that agree remarkably well with the experimental measurements. Agreement between theory and experiment is the best for smaller nanocrystal sizes; as their size increases, departure from spherical shape becomes more pronounced and the interaction with the substrate increases, effects not captured in this simple theoretical picture.

Cathodoluminescence (CL) was used to probe the local density of states of the nanocrystals, giving significant insight into the localization of the plasmon modes ${ }^{22}$ (Figure 3). 
For both sizes shown, the truncated bipyramids and icosahedra exhibit a 3-fold symmetric dipole response (Figure 3b). This is consistent with our theoretical simulations, shown in Figure 3c, where we calculate the far-field emission produced by the nanocrystals when excited by a single dipole placed at different positions on a plane $5 \mathrm{~nm}$ above the nanocrystal (simulating the electron excitation beam). These simulations were obtained by solving Maxwell's equations using a finite difference time-domain numerical solver (Lumerical).
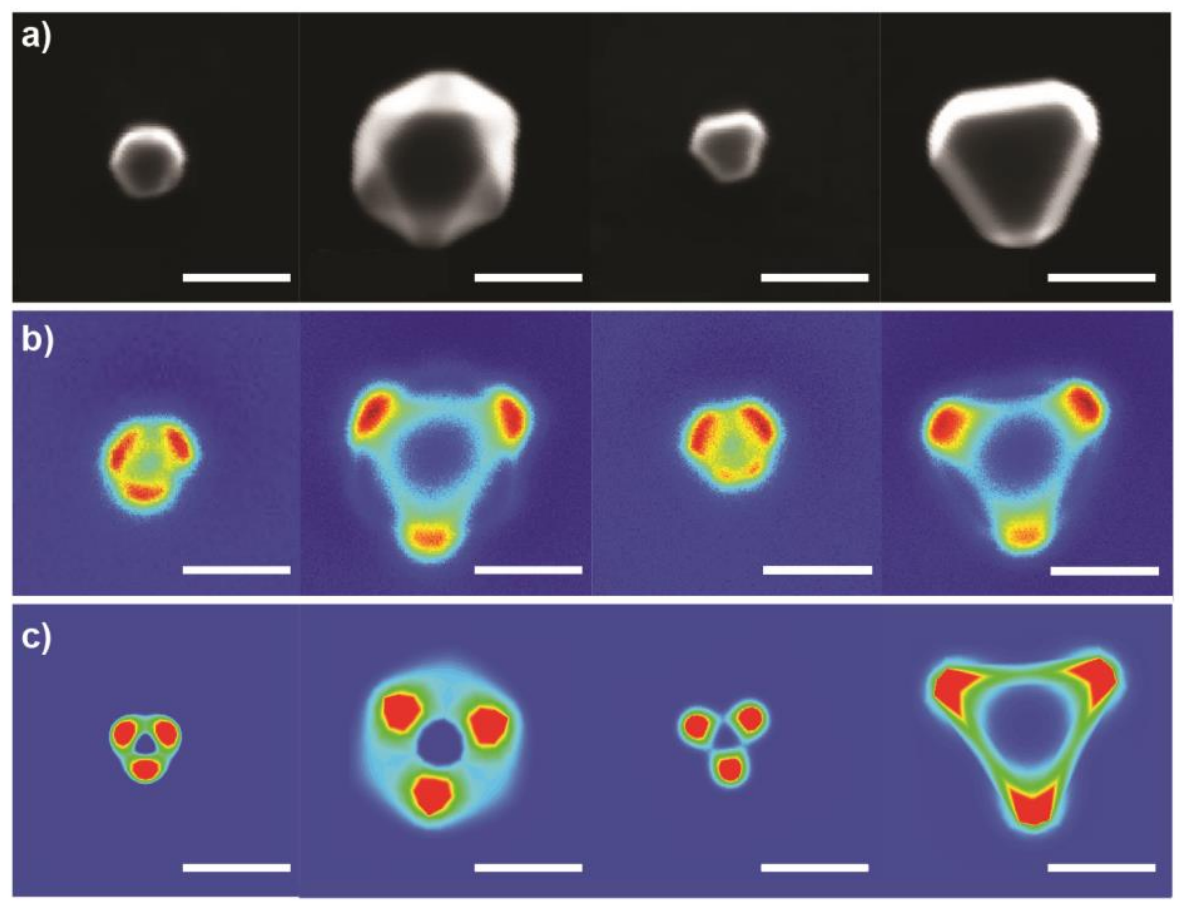

Figure 3. Cathodoluminescence images of the smallest and largest particles reveal similar charge density plots for varying sizes and shapes. a) SEM images of 70 and $155 \mathrm{~nm}$ icosahedra and 65 and $155 \mathrm{~nm}$ truncated trigonal bipyramids (right to left). b) Cathodoluminscence reveals threefold symmetry mode between $1.77 \mathrm{eV}$ and $3.54 \mathrm{eV}$. c) Calculated cathodoluminscence images simulated using a single dipole excitation. Scale bar $=100 \mathrm{~nm}$.

We also used electron energy-loss spectroscopy and imaging to characterize the Al nanocrystal plasmon modes; in this case, both the dark and the bright plasmon modes of the nanocrystal become accessible (Figure 4). ${ }^{21}$ 

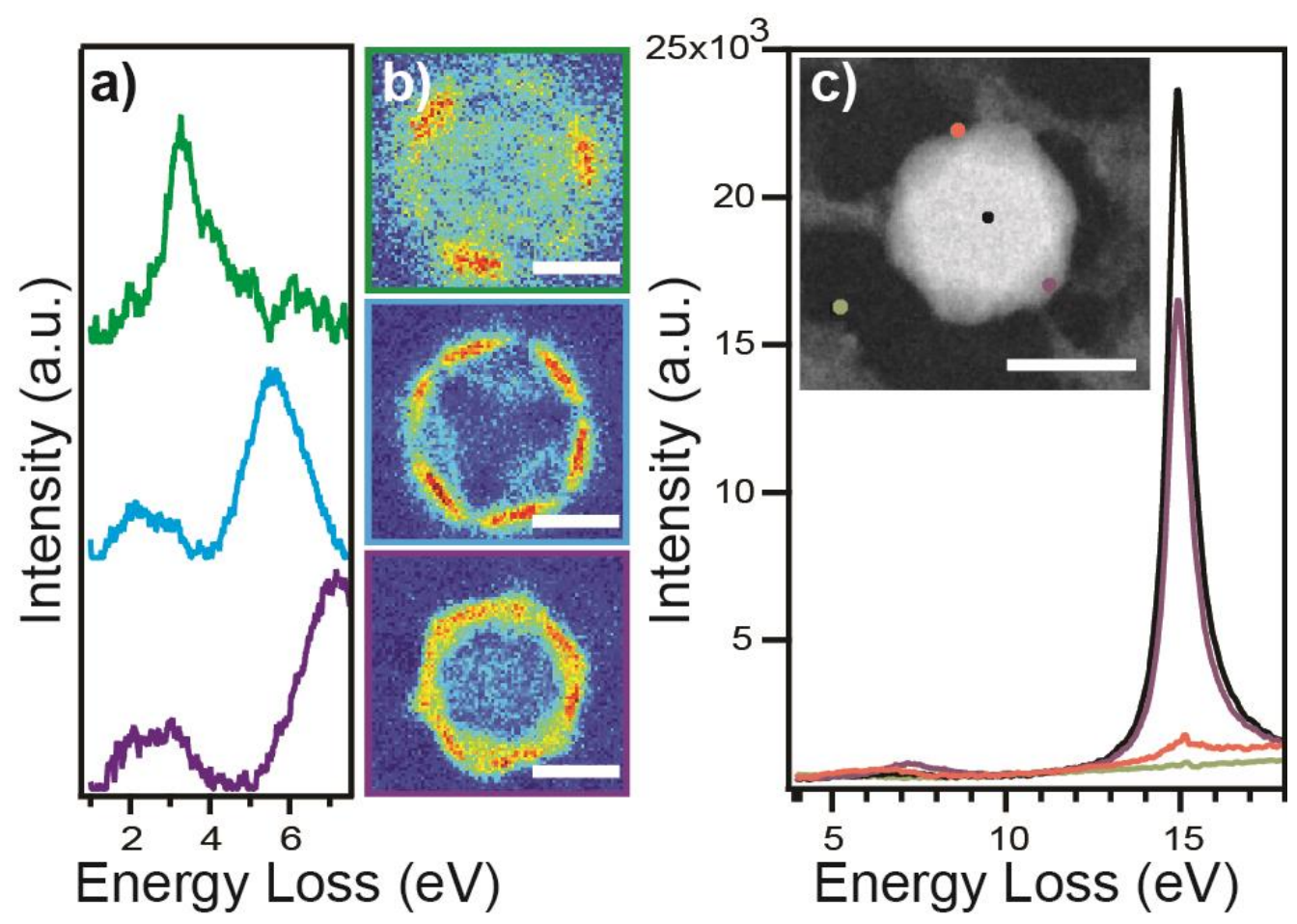

Figure 4. Electron-energy loss spectroscopy (EELS) map of $150 \mathrm{~nm}$ icosahedral nanocrystal. a) EELS spectral components extracted by non-negative matrix factorization. b) EELS images $($ Scale bar $=50 \mathrm{~nm})$ representing the spatial distribution of the plasmon modes corresponding to the spectra in (a). c) EELS of an Al nanocrystal at multiple points, as displayed in the STEM image (inset). Scale bar $=100 \mathrm{~nm}$.

Strong plasmon resonances that were well-defined both spatially and spectrally were observed consistently in all single nanocrystals studied. For the representative $165 \mathrm{~nm}$ diameter Al nanocrystal studied here, three prominent modes were found: the lowest energy at $3.3 \mathrm{eV}$ and intermediate energy modes at 5.5 and $7.1 \mathrm{eV}$ (Figure 4a). EELS images were obtained at spectral windows corresponding to these three mode energies (Figure $4 \mathrm{~b}$ ), which provided sufficient bandwidth to allow the use of non-negative matrix factorization, a powerful and assumption-free modal decomposition approach, to generate the nanocrystal image ${ }^{23,24}$. The nanocrystal images consistently display 3-fold symmetry, consistent with the CL images. The field intensity for the 
lowest energy dipolar $3.3 \mathrm{eV}$ plasmon mode (Figure $4 \mathrm{a}$, top) is strongly localized at the tips of the particle, similar to the lower energy modes of a nanocube. ${ }^{23,24}$ The $5.5 \mathrm{eV}$ mode (Figure 4a, center) is a quadrupolar surface plasmon resonance, with the field intensity localized at the faces of the structure. The $7.1 \mathrm{eV}$ mode (Figure 4a, bottom) is also localized at the nanoparticle surface and is consistent with a dark higher order octupolar plasmon mode that is slightly redshifted due to the presence of the thin oxide layer at the surface of the nanocrystal. The presence of this oxide layer on all the particles is further confirmed by oxygen mapping via EELS (Figure S3). This thin layer does not significantly alter the plasmonic properties of the nanoparticle and does not prevent efficient plasmon excitation, either in optical spectroscopy or electron-based techniques ${ }^{4}$. In addition to mapping core elemental transitions and surface plasmons, EELS also provides a handle on the bulk plasmon, which is particularly pronounced in $\mathrm{Al}$ (Figure 4c). The very strong mode observed around $14.9 \mathrm{eV}$ is indeed characteristic of the bulk plasmon resonance of $\mathrm{Al}$, confirming the nanoparticle composition. In Figure 4c, the purple and black points are away from the oxide edge and they show large Al bulk plasmon mode signals. The red and black locations probe the surface oxide layer and background, respectively, and show no Al bulk plasmon mode signal. Combining these results with the oxygen mapping (Figure S3) tells us these are Al NCs with an aluminum metal core and an oxide shell.

These uniform, robust, and air-stable nanocrystals are likely to be useful in many of the applications currently being pursued with gold and silver nanoparticles, including chemical sensing, surface-enhanced spectroscopies, and plasmonic materials and devices. In addition, they provide a far more sustainable alternative to gold and silver nanoparticles currently in widespread use, making them attractive for large-scale or large-area applications not considered practical previously, such as smart windows or displays, even paints and coatings. These new 
materials may replace organic pigments in applications where conditions such as long-term UV exposure may prove limiting to material lifetimes.

\section{ASSOCIATED CONTENT}

Supporting Information. Histograms of Al NCs represented in Figure 1 and TEM images of Al NCs washed in THF and IPA. EELS map of oxygen on an Al NC. This material is available free of charge via the Internet at http://pubs.acs.org.

\section{AUTHOR INFORMATION}

\section{Corresponding Author}

*E-mail: halas@rice.edu

\section{Funding Sources}

This work was supported by the Robert A. Welch Foundation under Grants C-1220 (N. J. H), C1222 (P.N), and C-0976 (K. H. W.); the Air Force Office of Scientific Research (AFOSR) FA9550-10-1-0469, NSF MRI, the Army's in-house laboratory independent research program, and the Army Research Office. A. M. acknowledges financial support from the Welch foundation through the J. Evans Attwell-Welch Postdoctoral Fellowship Program of the Smalley Institute of Rice University (Grant No. L-C-004).

\section{ACKNOWLEDGMENT}

The authors would like to thank Bob Y. Zheng, Amanda M. Goodman, Sandra W. Bishnoi, Desmond E. Schipper, and Felix Berg for productive discussions.

\section{REFERENCES}


(1) Alayoglu, S.; Nilekar, A. U.; Mavrikakis, M.; Eichhorn, B. Nat. Mater. 2008, 7, 333-338.

(2) Atwater, H. a; Polman, A. Nat. Mater. 2010, 9, 205-213.

(3) Hu, J.; Chen, L.; Lian, Z.; Cao, M.; Li, H.; Sun, W.; Tong, N. J. Phys. Chem. C 2012, 116, 15584-15590.

(4) Knight, M. W.; King, N. S.; Liu, L.; Everitt, H. O.; Nordlander, P. ACS Nano 2014, 8, 834-840.

(5) Knight, M. W.; Liu, L.; Wang, Y.; Brown, L.; Mukherjee, S.; King, N. S.; Everitt, H. O.; Nordlander, P.; Halas, N. J. Nano Lett. 2012, 12, 6000-6004.

(6) Linic, S.; Christopher, P.; Ingram, D. B. Nat. Publ. Gr. 2011, 10, 911-921.

(7) Schade, M.; Fuhrmann, B.; Bohley, C.; Schlenker, S.; Sardana, N.; Schilling, J.; Leipner, H. S. J. Appl. Phys. 2014, 115, 084309.

(8) Eghtedari, M.; Oraevsky, A.; Copland, J. A.; Kotov, N. A.; Conjusteau, A.; Motamedi, M. Nano Lett. 2007, 7, 1914-1918.

(9) Lohse, S. E.; Burrows, N. D.; Scarabelli, L.; Liz-marza, L. M.; Murphy, C. J. Chem. Mater. 2014, 26, 34-43.

(10) Brien, M. N. O.; Jones, M. R.; Brown, K. A.; Mirkin, C. A. J. Am. Chem. Soc. 2014, 136, 7603-7606.

(11) Chung, S. W.; Guliants, E. a; Bunker, C. E.; Hammerstroem, D. W.; Deng, Y.; Burgers, M. a; Jelliss, P. a; Buckner, S. W. Langmuir 2009, 25, 8883-8887.

(12) Shahravan, A.; Desai, T. G.; Matsoukas, T. ACS Appl. Mater. Interfaces 2014, 6, 79427947.

(13) Zidan, R. a; Takara, S.; Hee, A. G.; Jensen, C. M. J. Alloys Compd. 1999, 285, 119-122.

(14) Gavrilenko, V. V; Chekulaeva, L. A.; Zakharkin, L. I.; Khimicheskaya, S. Bull. Acad. Sci. USSR Div. Chem. Sci. 1977, 26, 1131-1134.

(15) Arora, N.; Jagirdar, B. R. J. Mater. Chem. 2012, 22, 9058.

(16) Jouet, R. J.; Warren, A. D.; Rosenberg, D. M.; Bellitto, V. J.; Park, K.; Zachariah, M. R. Chem. Mater. 2005, 17, 2987-2996.

(17) Lewis, W. K.; Rosenberger, A. T.; Gord, J. R.; Crouse, C. A.; Harruff, B. A.; Fernando, K. A. S.; Smith, M. J.; Phelps, D. K.; Spowart, J. E.; Guliants, E. A.; Bunker, C. E. 2010, 114, 6377-6380.

(18) Meziani, M. J.; Bunker, C. E.; Lu, F.; Li, H.; Wang, W.; Guliants, E. a; Quinn, R. a; Sun, Y.-P. ACS Appl. Mater. Interfaces 2009, 1, 703-709.

(19) Fernando, K. A. S.; Smith, M. J.; Harruff, B. A.; Lewis, W. K.; Guliants, E. A.; Bunker, C. E. J. Phys. Chem. C 2009, 113, 500-503.

(20) N. H. Chou, X. Ke, P. Schiffer, R. E. S. J. Am. Chem. Soc. 2008, 95S, 8140-8141.

(21) Nelayah, J.; Kociak, M.; Stéphan, O.; García de Abajo, F. J.; Tencé, M.; Henrard, L.; Taverna, D.; Pastoriza-Santos, I.; Liz-Marzán, L. M.; Colliex, C. Nat. Phys. 2007, 3, 348353.

(22) García de Abajo, F. J. Rev. Mod. Phys. 2010, 82, 209-275.

(23) Nicoletti, O.; de la Peña, F.; Leary, R. K.; Holland, D. J.; Ducati, C.; Midgley, P. a. Nature 2013, 502, 80-84.

(24) Ringe, E.; McMahon, J. M.; Sohn, K.; Cobley, C.; Xia, Y.; Huang, J.; Schatz, G. C.; Marks, L. D.; Van Duyne, R. P. J. Phys. Chem. C 2010, 114, 12511-12516. 
Table of Contents Graphic:

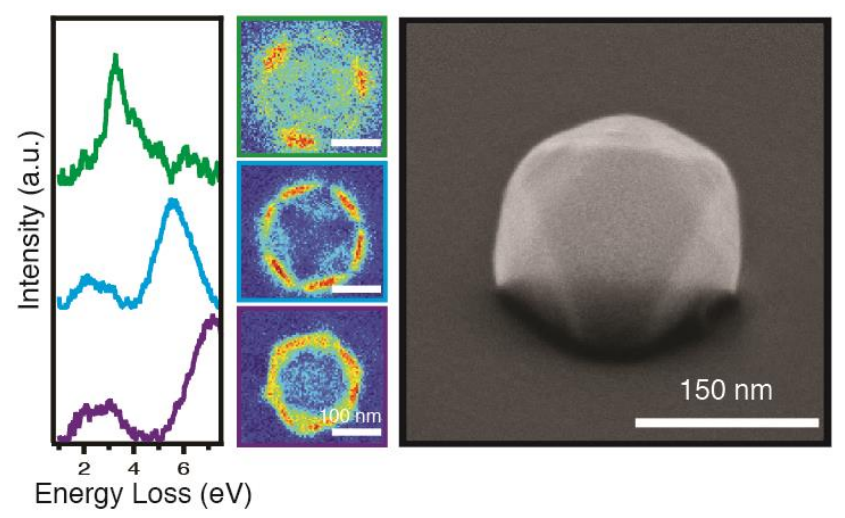

